\title{
Size Selective Synthesis of Superparamagnetic Nanoparticles in Thin Fluids under Continuous Flow Conditions**
}

\author{
By Suk Fun Chin, K. Swaminathan Iyer, Colin L. Raston,* and Martin Saunders
}

Continuous flow spinning disc processing (SDP), which has extremely rapid mixing under plug flow conditions, effective heat and mass transfer, allowing high throughput with low wastage solvent efficiency, is effective in gaining access to superparamagnetic $\mathrm{Fe}_{3} \mathrm{O}_{4}$ nanoparticles at room temperature. These are formed by passing ammonia gas over a thin aqueous film of $\mathrm{Fe}^{2+/ 3+}$ which is introduced through a jet feed close to the centre of a rapidly rotating disc (500 to $2500 \mathrm{rpm}$ ), the particle size being controlled with a narrow size distribution over the range $5 \mathrm{~nm}$ to $10 \mathrm{~nm}$, and the material having very high saturation magnetizations, in the range $68-78 \mathrm{emu} \mathrm{g}^{-1}$.

\section{Introduction}

Superparamagnetic magnetite $\left(\mathrm{Fe}_{3} \mathrm{O}_{4}\right)$ nanoparticles are important for a diverse range of applications such as, magnetic resonance imaging, targeted drug delivery and magnetic separation. ${ }^{[1]}$ These applications require the nanoparticles to be superparamagnetic with sizes smaller than $20 \mathrm{~nm}$, and with samples having a narrow size distribution to ensure the particles have uniform physical and chemical properties. ${ }^{[2]}$ The synthetic protocols for gaining access to such particles range from thermal decomposition of organometallic compounds in high-boiling organic solvents in the presence of surfactants ${ }^{[3]}$ to synthesis involving the use of reverse micelles, where surfactant-stabilized water-in-oil-emulsions control the shape and size of the $\mathrm{Fe}_{3} \mathrm{O}_{4}$ nanoparticles. ${ }^{[4]}$ However, these methods cannot be applied to large-scale and economic production as they require expensive or toxic organic reagents, and sequential and lengthy processing steps. A synthesis route for ultra-large-scale production of monodispersed $\mathrm{Fe}_{3} \mathrm{O}_{4}$ nanocrystals was recently reported. ${ }^{[5]}$ However, the particles were synthesized in the presence of oleic acid, which makes the particles only dispersible in organic solvents and this limits their bioavailability and hence their medical applications.

[*] Prof. C. L. Raston, S. F. Chin, Dr. K. S. lyer Center for Strategic Nano-Fabrication

School of Biomedical, Biomolecular and Chemical Sciences

The University of Western Australia

Crawley, W.A. 6009 (Australia)

E-mail: clraston@chem.uwa.edu.au

Dr. M. Saunders

Center for Microscopy, Characterization and Analysis,

The University of Western Australia

Crawley, W.A. 6009 (Australia)

[**] The authors are grateful for the financial support for this work by the Australian Research Council, The University of Western Australia, and The University of Malaysia Sarawak. The microscopy analysis was carried out using facilities at the Centre for Microscopy, Characterization and Analysis, The University of Western Australia, which are supported by University, State and Federal Government funding. Supporting Information is available online from Wiley InterScience or from the authors.
The most common cost effective and convenient way to synthesize $\mathrm{Fe}_{3} \mathrm{O}_{4}$ nanoparticles is by co-precipitating ferrous and ferric salt solutions with a base, such as aqueous $\mathrm{NaOH}$ or $\mathrm{NH}_{4} \mathrm{OH}^{[6]}$ However, the size distribution of the $\mathrm{Fe}_{3} \mathrm{O}_{4}$ nanoparticles produced using this method is normally very broad. Consequently, the downstream purification and isolation process is more expensive and is time and energy intensive. Furthermore, scale-up of this method using conventional reactors can be problematic given the inhomogeneous agitation and areas of localized $\mathrm{pH}$ variations, resulting in the precipitation of non-magnetic iron oxides. ${ }^{[7]}$

Accordingly, there is a growing demand in the nanotechnology industries for processes that promise to make dramatic improvements in the design and performance of the manufacturing equipment involved. The concept of "Process Intensification" offers alternative routes alleviating the obstacles of the relaxed fluid dynamic regime associated with conventional batch processes. Herein we demonstrate the successful synthesis of $\mathrm{Fe}_{3} \mathrm{O}_{4}$ nanoparticles via co-precipitation using $\mathrm{NH}_{3}$ gas as a base source using spinning disc processing (SDP) under scalable and continuous flow conditions. To our knowledge, this is the first use of $\mathrm{NH}_{3}$ gas as a precipitating agent to make $\mathrm{Fe}_{3} \mathrm{O}_{4}$ nanoparticles in a thin fluid film. The technology offers a realistic route towards large scale synthesis of $\mathrm{Fe}_{3} \mathrm{O}_{4}$ nanoparticles with precise control within the $10 \mathrm{~nm}$ size range.

The hydrodynamics of film flow over a spinning disc is important in controlling the reactions. The demand for intensified processing for which SDP is a subset has led to the design and development of a range of reactors that offer operating conditions with rapid heat and mass transfer under continuous flow conditions with residence times reduced to seconds rather than minutes or hours. SDP offers a novel avenue for intensified nanotechnology via exploitation of high centrifugal acceleration to generate thin films providing rapid heat and mass transfers. ${ }^{[8]}$ SDP is a form of process intensification where all reacting components are exposed to the same conditions, in contrast to traditional batch technology where conditions can vary across the dimensions of the vessel. ${ }^{\left[{ }^{[]}\right.}$The geometry and key elements of a SDP are illustrated in 